\title{
Comparisons of eccentric knee flexor strength and asymmetries across elite, sub-elite and school level cricket players
}

Wade J Chalker, Anthony J Shield, David A Opar, Justin W.L Keogh

Background. There has been a continual increase in injury rates in cricket, with hamstring strain injuries (HSIs) being the most prominent. Eccentric knee flexor weakness and bilateral asymmetries are major modifiable risk factors for future HSIs. However, there is a lack of data relating to eccentric hamstring strength in cricket at any skill level. The objective of this study was to compare eccentric knee flexor strength and bilateral asymmetries in elite, sub-elite and school level cricket players; and to determine if playing position and limb role influenced these eccentric knee flexor strength indices. Methods. Seventy four male cricket players of three distinct skill levels performed three repetitions of the Nordic hamstring exercise on the experimental device. Strength was assessed as the absolute and relative mean peak force output for both limbs, with bilateral asymmetries. Differences in mean peak force outputs between skill level and playing positions were measured. Results. There were no significant differences between elite, sub-elite and school level athletes for mean peak force and bilateral asymmetries of the knee flexors. There were no significant differences observed between bowlers' and batters' mean peak force and bilateral asymmetries. There were no significant differences between front and back limb mean peak force outputs. Discussion. Skill level, playing position and limb role appeared to have no significant effect on eccentric knee flexor strength and bilateral asymmetries. Future research should seek to determine whether eccentric knee flexor strength thresholds are predictive of HSIs in cricket and if specific eccentric knee flexor strengthening can reduce these injuries. 
1 Comparisons of Eccentric Knee Flexor Strength and Asymmetries Across Elite, Sub-Elite

2 and School Level Cricket Players

3 Wade J. Chalker, BSpSci ${ }^{1}$

4 Anthony J. Shield, $\mathrm{PhD}^{2}$

5 David A. Opar, $\mathrm{PhD}^{3}$

6 Justin W.L. Keogh, $\mathrm{PhD}^{1,4,5}$

$7 \quad{ }^{1}$ Faculty of Health Sciences and Medicine, Bond University, Gold Coast, Australia

$8{ }^{2}$ School of Exercise and Nutrition Sciences, Queensland University of Technology, Brisbane,

9 Australia

$10{ }^{3}$ Faculty of Health Sciences, Australian Catholic University, Melbourne, Australia

$11{ }^{4}$ Sports Performance Research Centre New Zealand, Auckland University of Technology, 12 Auckland, New Zealand

135 Cluster for Health Improvement, Faculty of Science, Health, Education and Engineering, 14 University of the Sunshine Coast

15 Address correspondence to Justin W.L. Keogh, Faculty of Health Sciences and Medicine, Bond

16 University, University Drive, Robina, Gold Coast, Australia, 4226. E-mail: jkeogh@bond.edu.au 


\section{Introduction}

18 Cricket is a team sport involving a side of 11 players with specialist batters, bowlers and one

19 wicket-keeper. Originating from test match cricket which was typically played over 5 days

20 between two teams with unlimited overs, limited overs cricket ( 50 overs per team) and Twenty20

21 (20 overs per team) games lasting 7-8 hours and 3 hours respectively are also now widely played

22 (Cannonier et al. 2013). As a result of these new formats, elite level cricket players are now

23 playing more cricket each year, with the intensity of these games also appearing to increase (Frost

24 \& Chalmers 2014).

Injury rates appear to be increasing for elite cricketers in many countries, with hamstring strain injuries (HSIs) often the most commonly occurring injury (Frost \& Chalmers 2014; Orchard et al. 2002; Orchard et al. 2011; Orchard et al. 2006; Stretch 2003). Less injuries are reported for junior compared to senior players, with most junior injuries associated with ball contact (Finch et al. 2010). Elite pace bowlers may be at higher risk of HSIs than batters and wicket-keepers (Orchard et al. 2011). The pace bowler's greater HSI risk may reflect their greater total running and sprint distances (Petersen et al. 2010) as well as the intense loads placed on the front leg during ball release when bowling, whereby the knee flexors act to rapidly decelerate the total body's forward momentum resulting from their run-up. Player position (batter or bowler) and limb dominance (front or back leg) during batting, bowling and throwing may therefore have an impact on HSI risk in cricket. However, the symmetry, or lack thereof of knee flexor function is yet to be documented in cricket, unlike other sports (Bourne et al. 2015; Opar et al. 2015). Throughout this paper, knee flexors refer to the muscle action of knee flexion resulting from the involvement of

38 the hamstrings (bicep femoris long head and short, semitendinosus and semimembranosus) muscles assisted by the gastrocnemius. When the phrase hamstrings is used in the manuscript, it 
40 is generally used anatomically in relation to any injury to the bicep femoris, semitendinosus or

41 semimembranosus muscle.

42 Risk factors that have been associated with HSIs in the wider sports medicine literature include;

43 increased age, previous hamstring injuries, lack of hamstring flexibility, muscle fatigue and

44 hamstring muscle weakness or asymmetry (Brooks et al. 2006; Croisier et al. 2002; Croisier et al.

45 2008; Gabbe et al. 2006; Jonhagen et al. 1994; Opar et al. 2012; Orchard et al. 1997; Verrall et al.

46 2001). In particular, eccentric knee flexor strength deficits and bilateral eccentric knee flexor

47 strength asymmetries have been linked to an increased risk of HSIs (Brooks et al. 2006; Croisier

48 et al. 2008; Opar et al. 2012; Orchard et al. 1997). A novel field testing device has recently been

49 developed to measure eccentric knee flexor strength and strength asymmetries via uniaxial load

50 cells during the Nordic hamstring exercise (NHE) (Opar et al. 2013; Opar et al. 2015). Elite

51 Australian football players with eccentric knee flexor strength below $256 \mathrm{~N}$ and $279 \mathrm{~N}$ at the start

52 and end of pre-season training, respectively, have approximately a 3 to 4 fold increased risk of

53 sustaining a HSI (Opar et al. 2015), further confirming the importance of eccentric knee flexor

54 strength in the prevention of HSIs. Additionally, the elevated probability of sustaining a HSI for

55 older athletes or those with a previous HSI can be offset with greater eccentric knee flexor

56 strength (Opar et al. 2015). Recent studies using the novel field testing device in elite Australian

57 football and rugby union present somewhat contrasting results on the predictive ability of

58 between limb asymmetries and eccentric knee flexor strength for HSIs. Elite Australian football

59 players with a between limb strength asymmetry of $\geq 10 \%$ did not have an increased risk of

60 sustaining a HSI (Opar et al. 2015). This contrasts with a study involving rugby union players

61 where a between limb strength asymmetry $\geq 15 \%$ and $\geq 20 \%$ had an increased risk of sustaining a

62 HSI by 2.4 and 3.4 fold respectively (Bourne et al. 2015). Such studies suggest that between limb

63 strength asymmetries may be an issue in some sports but not others. Additional studies have 
64 found that asymmetries greater than 10\%, 8\% and 15\% for track and field athletes, Australian

65 football players and soccer players respectively, increase the risk of occurrence of a HSI (Croisier

66 et al. 2008; Heiser et al. 1984; Opar et al. 2012; Orchard et al. 1997).

67 Given the lack of data relating to eccentric knee flexor strength in cricket at any level, the

68 asymmetrical demands of cricket batting, bowling and throwing as well as the increasing

69 prevalence of HSIs in elite cricket, the primary purpose of this investigation was to measure

70 eccentric knee flexor strength and bilateral asymmetries in elite, sub-elite and school level cricket

71 players. The secondary purpose was to determine if playing position and limb role influenced

72 eccentric knee flexor strength or the magnitude of asymmetries.

\section{Materials \& Methods}

\section{Participants}

75 Seventy four male cricket players (16 elite level, 32 sub-elite level and 26 school level) with at

76 least two years of experience in the sport provided written informed consent before participating

77 in the study. Athletes with a previous and/or current lower limb injury that had not yet been fully

78 rehabilitated were not included in the study. Ethical approval was granted by the Bond University

79 Human Research Ethics Committee (RO1824).

80 Research Design

81 A cross sectional comparative study design was used with all testing performed at the start of pre-

82 season training before the commencement of a training session, at the start of the training week.

83 This was to be consistent with previous studies using the experimental device (Bourne et al.

84 2015; Opar et al. 2015) and to control for any potential differences in training that occur between

85 the playing groups over the course of the season. All participants completed a familiarisation

86 session, in which they performed two sets of three repetitions of the NHE on the novel field 
87 testing device while being provided with coaching cues for correct technique. One week later,

88 they were reassessed to determine eccentric knee flexor strength and bilateral asymmetries on a

89 novel field testing device with established reliability (Opar et al. 2013). Prior to the main testing

90 session, a submaximal warm-up set of the NHE of three repetitions (first repetition at $\sim 50 \%$ of

91 maximum perceived exertion, second repetition at $\sim 70 \%$ of maximum perceived exertion and the

92 third repetition at $\sim 90 \%$ of their maximum perceived exertion) was performed before the athletes

93 completed one set of three maximal efforts with one minute rest between sets and 15 seconds

94 inter-repetition rest.

\section{Eccentric Knee Flexor Strength Assessment}

96 Strength was assessed as the peak force output on the field testing device previously assessed for 97 reliability (Opar et al. 2013). All participants were asked to kneel on a padded board with their 98 ankles secured immediately superior to the lateral malleolus. Separate securing braces were 99 attached to custom made uniaxial load cells (Delphi Force Measurements) fitted with wireless 100 data acquisition capabilities (Mantracourt Electronics Ltd). Ankle braces were secured to the 101 testing device via a pivot system to ensure force was always measured through the long axis of 102 the load cells. Athletes were instructed to gradually lean forward at the slowest possible speed 103 while maximally resisting the tendency to fall with both limbs, keeping the trunk and hips in a 104 neutral position throughout the movement with hands placed across the chest (Opar et al. 2013). 105 Verbal encouragement and technique coaching was provided throughout each repetition to 106 promote maximal efforts.

\section{Data Analysis}

108 Force data for both left and right limbs during the NHE were logged to a personal computer at $10950 \mathrm{~Hz}$ through a wireless receiver (Mantracourt Electronics Ltd). Mean peak force (N) was 110 calculated for both limbs for the three maximal repetitions. The relative peak force was calculated 
111 by dividing the mean peak force $(\mathrm{N})$ by the participant's body mass $(\mathrm{kg})$ so to account for any

112 differences on body-mass between participant groups. The between limb imbalance in eccentric

113 knee flexor force was calculated as a left:right limb ratio of the mean peak force as

114 recommended, using log transformed raw data followed by back transformation (Impellizzeri et

115 al. 2008).

116 Statistical Analysis

117 All statistical analysis was performed using SPSS version 20.0 (IBM corporation). Means and 118 standard deviations (SD) of age, height, body weight, absolute and relative eccentric knee flexor 119 strength and eccentric knee flexor strength imbalances are presented. If data was not normally 120 distributed, as assessed by Shapiro-Wilks test for normality, log transformation was performed.

121 Log transformed data was then back transformed to represent true values. Data was compared 122 between all three playing groups (elite, sub-elite and school) and further compared based on their 123 self-nominated playing specialisation (bowler or batter) with Cohen $d$ values provided. Small 124 effect sizes were classified as $>0.2$, moderate effect size $>0.5$ and a large effect size $\geq 0.8$. One 125 way analysis of variance with a Tukey post hoc test was used to compare mean peak force output 126 (combined average of left and right limbs) and limb asymmetry between the three groups (elite, 127 sub-elite and school). An independent t-test compared the mean peak force and limb asymmetry 128 between playing positions (bowlers and batters). Paired t-tests were performed comparing front 129 and back leg mean peak force outputs, where front leg was considered the leg most forward at the 130 point of ball release when bowling and throwing and in the normal batting stance for batters. 131 Within-session variability of peak force and limb asymmetry were quantified via the coefficient 132 of variation, calculated for all three groups based on the three maximal repetitions. Significance 133 was set at $\mathrm{P}<0.05$. 
134

135

136

137

138

139

140

141

142

143

144

145

146

147

148

149

150

151

152

153

154

155

\title{
Results
}

The physical characteristics of each skill level and playing position are described in Table 1. Increases in skill level were associated with significantly increased player age, height and weight.

\author{
Insert Table 1 here
}

There were no significant differences between elite, sub-elite and school level athletes for absolute mean peak force (elite vs sub-elite $d=0.07$; sub-elite vs school $d=0.32$; elite vs school $d=0.42 ; \mathrm{P}>0.05$ ), relative mean peak force (elite vs sub-elite $d=-0.10$; sub-elite vs school $d=$ -0.42 ; elite vs school $d=-0.55 ; \mathrm{P}>0.05$ ) and bilateral asymmetries (elite vs sub-elite $d=-0.34$; sub-elite vs school $d=0.21$; elite vs school $d=-0.11 ; \mathrm{P}>0.05) .24$ athletes $(32.4 \%)$ had bilateral limb asymmetries $\geq 15 \%$, and $14(18.9 \%)$ of these athletes had asymmetries $\geq 20 \%$. Furthermore, Table 1 indicates there were no significant differences observed between bowler's and batter's absolute and relative mean peak force $(d=-0.14 ; \mathrm{P}>0.05)$ and bilateral asymmetries $(d=0.04$; $\mathrm{P}>0.05)$. Of the 24 athletes with limb asymmetries $\geq 15 \%, 14(33.3 \%)$ were bowlers and 10 were batters $(31.3 \%)$. Nine bowlers $(21.4 \%)$ and 5 batters $(15.6 \%)$ had limb asymmetries $\geq 20 \%$. There were no significant differences between front and back limb absolute mean peak force outputs $(299 \pm 79 \mathrm{~N}$ and $303 \pm 71 \mathrm{~N} ; d=-0.05 ; \mathrm{P}>0.05)$ and relative mean peak force $\left(3.83 \pm 1.03 \mathrm{~N} \mathrm{~kg}^{-1}\right.$ and $\left.3.84 \pm 0.84 \mathrm{~N} . \mathrm{kg}^{-1} ; d=-0.01 ; \mathrm{P}>0,05\right)$ (see Figure 1$)$

Insert Table 2 here

Insert Figure 1 here

\section{Discussion}

To the best of our knowledge, this is the first paper to measure eccentric knee flexor strength in cricket players of any level. As HSI rates are increasing in cricket (Frost \& Chalmers 2014; 
156 Orchard et al. 2011; Orchard et al. 2006), this study may present the first step in identifying the

157 potential role of eccentric knee flexor strength and bilateral asymmetries in predisposing cricket 158 players to such injury. The lack of any significant difference in eccentric knee flexor strength or 159 asymmetry between skill level was surprising. Elite players were expected to be stronger as they 160 should have greater experience in performing knee flexor strengthening exercises and they place 161 greater loads on their hamstrings as a result of their greater running and sprinting distances 162 (Petersen et al. 2010). There was only a small effect size between sub-elite and school level 163 athletes and a small to moderate effect size between elite and school level athletes for absolute 164 mean peak force. Relative mean peak force demonstrated a small to moderate effect size between 165 sub-elite and school level athletes and a moderate effect size between elite and school level 166 athletes. While cricket players possessed $\sim 80 \%$ of the eccentric knee flexor strength of rugby 167 union players of similar levels $(367 \mathrm{~N}, 389 \mathrm{~N}$ and $343 \mathrm{~N}$ for elite, sub-elite and under 19 athletes 168 respectively) (Bourne et al. 2015). The differences in eccentric knee flexor strength between skill 169 levels of rugby union players appears similar in magnitude to that of the current study in cricket, however, there was a significant difference in bilateral eccentric knee flexor force outputs

171 between the sub-elite and under 19 athletes in rugby union. Therefore, differences between the 172 cricket groups for mean peak force were expected considering the mean age of the school athletes $173(15.7 \pm 1$ year $)$ is lower than the rugby under 19 athletes. Due to the greater eccentric knee flexor 174 demands in elite compared to sub-elite and schoolboy sport, the results of this study and that of 175 Bourne et al. (2015) may suggest that additional specific eccentric knee flexor exercises may be 176 required in elite cricket and rugby to reduce their high prevalence of HSIs. Support for this 177 approach can be found in soccer where the addition of the NHE significantly reduced HSI injury rates (Arnason et al. 2008; Askling et al. 2003). Further, the fact that the school level athletes 179 possess greater relative mean peak force compared to elite and sub-elite athletes may explain why 180 the occurrences of HSIs in junior cricket are much lower compared to elite athletes who are 
181 playing and training under greater physical demands with a reduced capacity to protect the knee

182 flexors.

183 Compared to elite batters, elite pace bowlers may be more prone to HSIs due to the high-intensity 184 eccentric loads placed on the hamstrings of the front leg at ball release during bowling (Orchard 185 et al. 2011) and their greater sprinting distances (Petersen et al. 2010). This suggests that elite 186 pace bowlers may be the playing group most in need of additional eccentric knee flexor 187 strengthening exercises. Support for this view is provided by our findings which showed no 188 significant difference in eccentric knee flexor strength or bilateral asymmetries between bowlers 189 and batters. Additionally, bowlers were found to have less relative mean peak force compared to 190 that of batters $\left(3.74 \pm 0.97 \mathrm{~N} . \mathrm{kg}^{-1}\right.$ and $3.99 \pm 0.76 \mathrm{~N} \cdot \mathrm{kg}^{-1}$, respectively), where it is believed they 191 should maintain greater relative force as they are exposed to more eccentrically demanding knee 192 flexor actions. The relatively low average bilateral asymmetry for both playing positions suggests 193 that the game is not subjecting athletes to chronic limb asymmetries. However, greater 194 consideration for eccentric knee flexor strengthening exercises may need to be implemented for 195 bowlers at an elite and sub-elite level to help combat the high incidence rates of HSIs.

196 It is however unclear whether such additional eccentric knee flexor training should focus on 197 reducing bilateral asymmetries or increasing the strength of both knee flexors in elite cricketers. 198 Elite Australian football players with an eccentric knee flexor force output below $256 \mathrm{~N}$ at the 199 start of pre-season training were at an increased risk of sustaining a HSI (Opar et al. 2015), within 200 the 74 participants of this study, $21(28.4 \%)$ had a mean peak force output below 256N, and when 201 separated into playing positions, 14 bowlers $(33.3 \%)$ and 7 batters $(21.9 \%)$ had mean peak force 202 outputs below 256N. Elite Australian football players with a bilateral limb asymmetry of up to $20320 \%$, as measured on the same testing device as the current study, did not increase the risk of 204 sustaining a HSI (Opar et al. 2015). This contrasts with rugby union, where a 2.4 and 3.4 fold 
205 increase of HSI risk occurred with asymmetries of $\geq 15 \%$ and $\geq 20 \%$ respectively (Bourne et al.

206 2015). Results of the current study indicated similar mean levels of bilateral limb asymmetries for 207 bowlers (13.7\%) and batters (13.2\%) and across all three skill levels (11.5-15.1\%). This is further 208 supported with similar proportions of athletes at each skill level with bilateral limb asymmetries $209 \geq 15 \%(31.3 \%, 34.4 \%$ and $30.8 \%$ for elite, sub-elite and school level groups respectively). While 210 the contribution of knee flexor strength asymmetry on HSI rates seems to differ between sports, 211 further prospective studies involving cricket players throughout an entire season may be required 212 to better understand this possible relationship.

213 There was no significant difference between absolute and relative mean peak force for front limb 214 and back limb. It was expected that front limb would be stronger in both playing positions as 215 batters associate their front leg with the greatest amount of use and bowler's front legs are 216 associated with the greatest eccentric demands during the bowling action at ball release. The 217 exact causes for similar front and back limb forces across all participants are unknown and it may 218 come down to multiple influences that differ between each player.

219 To some extent the current study is limited by the sample sizes for each group, whereby larger 220 samples would allow greater certainty of the effect of skill level, playing position and leg 221 dominance on knee flexor limb strength and asymmetries. The cross-sectional nature of the data 222 also limits the inferences that can be drawn from the data. It is acknowledged that the time of 223 testing may not truly represent playing positions, however, it is believed as all athletes are 224 experienced in the game of cricket and their playing position any chronic asymmetries between 225 limbs would likely still be present at the start of pre-season training. 
226

227

228

229

230

231

232

233

234

235

236

237

238

239

240

241

242

243

244

245

246

247

248

249

250

251

252

253

254

\section{Conclusion}

There appears to be no significant differences between eccentric knee flexor strength or between limb strength asymmetries for elite, sub-elite and junior players during the NHE. Further, playing position (batter or bowler) or leg role (front or back) appears to have no significant effect on eccentric knee flexor strength and between limb strength imbalances for cricket players. Further research is required to prospectively monitor cricket players to determine if eccentric knee flexor strength or between limb strength asymmetries is a predictor of hamstring injury and if eccentric knee flexor strengthening exercise programs may reduce injury rates in cricket.

\section{References}

Arnason A, Andersen TE, Holme I, Engebretsen L, and Bahr R. 2008. Prevention of hamstring strains in elite soccer: an intervention study. Scand J Med Sci Sports 18:40-48. 10.1111/j.1600-0838.2006.00634.x

Askling C, Karlsson J, and Thorstensson A. 2003. Hamstring injury occurrence in elite soccer players after preseason strength training with eccentric overload. Scand J Med Sci Sports 13:244-250. 10.1034/j.1600-0838.2003.00312.x

Bourne MN, Opar DA, Williams MD, and Shield AJ. 2015. Eccentric Knee Flexor Strength and Risk of Hamstring Injuries in Rugby Union: A Prospective Study. Am J Sports Med. $10.1177 / 0363546515599633$

Brooks JHM, Fuller CW, Kemp SPT, and Reddin DB. 2006. Incidence, Risk, and Prevention of Hamstring Muscle Injuries in Professional Rugby Union. Am J Sports Med 34:1297-1306. $10.1177 / 0363546505286022$

Cannonier C, Panda B, and Sarangi S. 2013. 20-Over Versus 50-Over Cricket: Is There a Difference? J Sports Econ:1-24. 10.1177/1527002513505284

Croisier JL, Forthomme B, Namurois MH, Vanderthommen M, and Crielaard JM. 2002. Hamstring muscle strain recurrence and strength performance disorders. Am J Sports Med 30:199-203.

Croisier JL, Ganteaume S, Binet J, Genty M, and Ferret JM. 2008. Strength imbalances and prevention of hamstring injury in professional soccer players: a prospective study. Am J Sports Med 36:1469-1475. 10.1177/0363546508316764 
255 Finch CF, White P, Dennis R, Twomey D, and Hayen A. 2010. Fielders and batters are injured

256

257

258

259

260

261

262

263

264

265

266

267

268

269

270

271

272

273

274

275

276

277

278 too: a prospective cohort study of injuries in junior club cricket. J Sci Med Sport 13:489495. 10.1016/j.jsams.2009.10.489

Frost WL, and Chalmers DJ. 2014. Injury in elite New Zealand cricketers 2002-2008: descriptive epidemiology. Br J Sports Med 48:1002-1007. 10.1136/bjsports-2012-091337

Gabbe BJ, Bennell KL, and Finch CF. 2006. Why are older Australian football players at greater risk of hamstring injury? J Sci Med Sport 9:327-333. 10.1016/j.jsams.2006.01.004

Heiser TM, Weber J, Sullivan G, Clare P, and Jacobs RR. 1984. Prophylaxis and management of hamstring muscle injuries in intercollegiate football players. Am J Sports Med 12:368370.

Impellizzeri FM, Bizzini M, Rampinini E, Cereda F, and Maffiuletti NA. 2008. Reliability of isokinetic strength imbalance ratios measured using the Cybex NORM dynamometer. Clin Physiol Funct Imaging 28:113-119. 10.1111/j.1475-097X.2007.00786.x

Jonhagen S, Nemeth G, and Eriksson E. 1994. Hamstring injuries in sprinters. The role of concentric and eccentric hamstring muscle strength and flexibility. Am J Sports Med 22:262-266.

Opar DA, Piatkowski T, Williams MD, and Shield AJ. 2013. A Novel Device Using the Nordic Hamstring Exercise to Assess Eccentric Knee Flexors Strength: A Reliability and Retrospective Injury Study. J Orthop Sports Phys Ther. 2919 [pii] 10.2519/jospt.2013.4837

Opar DA, Williams MD, and Shield AJ. 2012. Hamstring strain injuries: factors that lead to injury and re-injury. Sports Med 42:209-226. 10.2165/11594800-000000000-00000

Opar DA, Williams MD, Timmins RG, Hickey J, Duhig SJ, and Shield AJ. 2015. Eccentric hamstring strength and hamstring injury risk in Australian footballers. Med Sci Sports Exerc 47:857-865. 10.1249/MSS.0000000000000465

Orchard J, James T, Alcott E, Carter S, and Farhart P. 2002. Injuries in Australian cricket at first class level 1995/1996 to 2000/2001. Br J Sports Med 36:270-274. 10.1136/bjsm.36.4.270

Orchard J, James T, Kountouris A, Blanch P, Sims K, and Orchard J. 2011. Injury Report 2011: Cricket Australia. Sport Health 29:16-29.

Orchard J, Marsden J, Lord S, and Garlick D. 1997. Preseason Hamstring Muscle Weakness Associated with Hamstring Muscle Injury in Australian Footballers. Am J Sports Med 25:81-85. 10.1177/036354659702500116 
287 288
Orchard JW, James T, and Portus MR. 2006. Injuries to elite male cricketers in Australia over a 10-year period. J Sci Med Sport 9:459-467. http://dx.doi.org/10.1016/j.jsams.2006.05.001

Petersen CJ, Pyne D, Dawson B, Portus M, and Kellett A. 2010. Movement patterns in cricket vary by both position and game format. J Sports Sci 28:45-52.

Stretch RA. 2003. Cricket injuries: a longitudinal study of the nature of injuries to South African cricketers. Br J Sports Med 37:250-253. 10.1136/bjsm.37.3.250

Verrall GM, Slavotinek JP, Barnes PG, Fon GT, and Spriggins AJ. 2001. Clinical risk factors for hamstring muscle strain injury: a prospective study with correlation of injury by magnetic resonance imaging. Br J Sports Med 35:435-440. 
296 Table 1. Physical characteristics of participants in each skill level and playing position.

\begin{tabular}{lcccc} 
& Elite $(\mathrm{n}=16)$ & Sub-elite $(\mathrm{n}=32)$ & School $(\mathrm{n}=26)$ & Combined $(\mathrm{n}=74)$ \\
\cline { 2 - 5 } Age & $24.5 \pm 4.5$ & $21.5 \pm 4.2^{*}$ & $15.7 \pm 1.0^{* \dagger}$ & $20.1 \pm 4.9$ \\
Height $(\mathrm{cm})$ & $186.2 \pm 9.5$ & $184.2 \pm 6.9$ & $178 \pm 7.2^{*}$ & $183.1 \pm 7.9$ \\
Weight $(\mathrm{kg})$ & $86.3 \pm 8.1$ & $83.1 \pm 10.3$ & $69.4 \pm 11.0^{* \dagger}$ & $79.0 \pm 12.3$
\end{tabular}

\begin{tabular}{lcc} 
& Bowlers $(\mathrm{n}=42)$ & Batters $(\mathrm{n}=32)$ \\
\cline { 2 - 3 } Age & $20.1 \pm 4.7$ & $20.1 \pm 5.2$ \\
Height $(\mathrm{cm})$ & $184.2 \pm 8.4$ & $181.7 \pm 7.1$ \\
Weight $(\mathrm{kg})$ & $80.3 \pm 13.0$ & $77.3 \pm 11.3$
\end{tabular}

$297 *$ Significantly different to elite level athletes

$298+$ Significantly different to sub-elite level athletes 
299 Table 2. Mean peak force outputs and relative mean peak force outputs with bilateral limb 300 asymmetries of knee flexors during the NHE for all three groups; elite, sub-elite and school level 301 and playing positions; bowlers and batters.

\begin{tabular}{lccc} 
& $\begin{array}{c}\text { Absolute Mean } \\
\text { Peak Force }(\mathrm{N})\end{array}$ & $\begin{array}{c}\text { Relative Mean Peak } \\
\text { Force }\left(\mathrm{N} . \mathrm{kg}^{-1}\right)\end{array}$ & $\begin{array}{c}\text { Bilateral } \\
\text { Asymmetry (\%) }\end{array}$ \\
\cline { 2 - 4 } & $313 \pm 67$ & $3.65 \pm 0.89$ & $11.5 \pm 8.6$ \\
Elite & $308 \pm 77$ & $3.74 \pm 0.96$ & $15.1 \pm 12.2$ \\
Sub-elite & $285 \pm 68$ & $4.11 \pm 0.77$ & $12.6 \pm 11.6$ \\
School & & & \\
& & & \\
Bowler & $297 \pm 77$ & $3.74 \pm 0.97$ & $13.7 \pm 10.3$ \\
Batter & $305 \pm 65$ & $3.99 \pm 0.76$ & $13.2 \pm 12.5$ \\
\hline
\end{tabular}

Data presented as mean \pm standard deviation. 


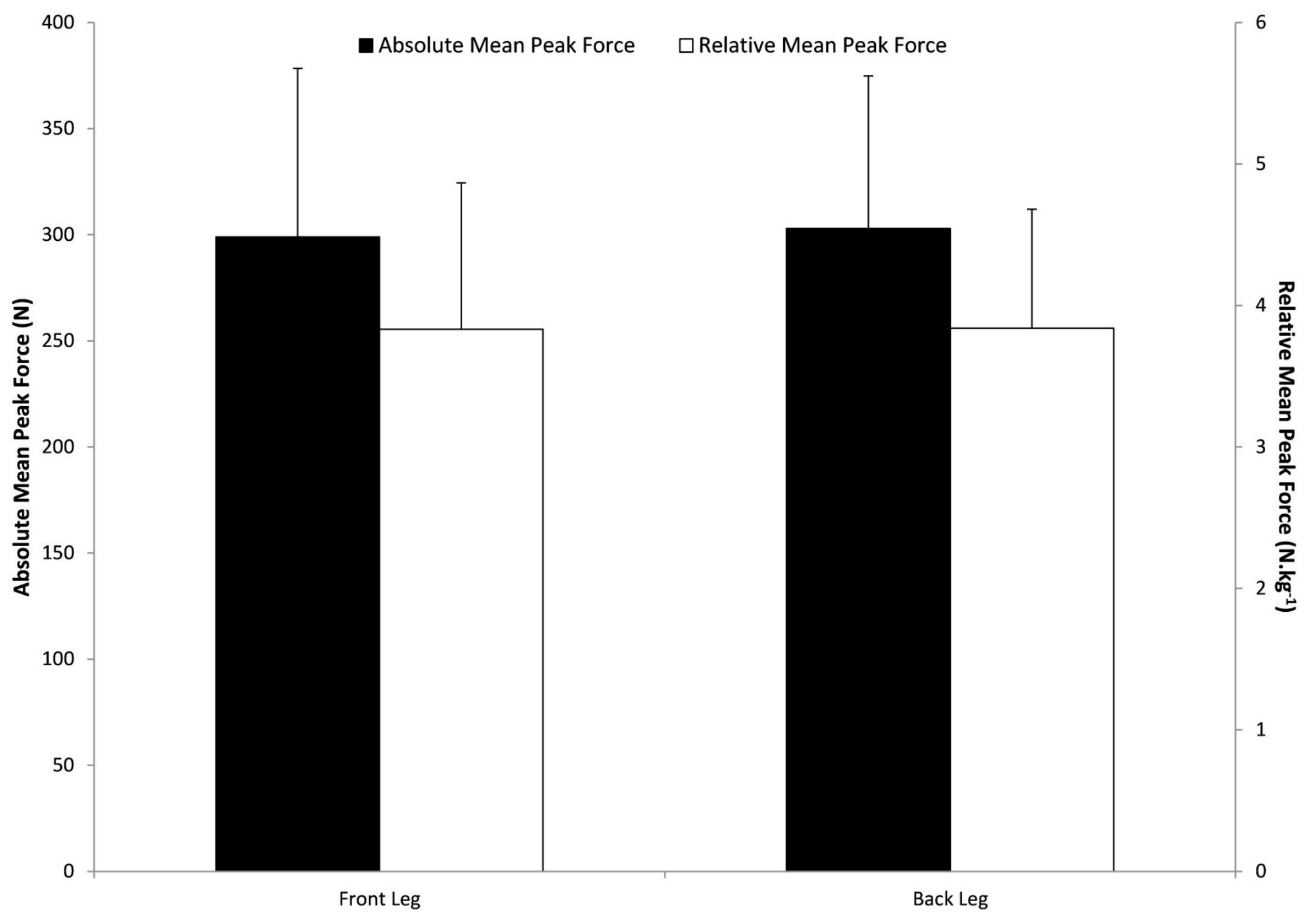

302 Figure 1. Mean peak force outputs (N) and relative mean peak force outputs $\left(\mathrm{N} \cdot \mathrm{kg}^{-1}\right)$ for 303 combined group $(n=74)$ comparing front leg to back leg. 